\title{
HOW TO INTERPRET AN ESTIMATED GLOMERULAR FILTRATION RATE (eGFR) IN 2020?
}

\author{
Marc De Broe ${ }^{1}$ and Pierre Delanaye ${ }^{2,3}$ \\ ${ }^{1}$ Laboratory of Physiopathology, University of Antwerp, Antwerpen, Belgium \\ ${ }^{2}$ Department of Nephrology-Dialysis-Transplantation, University of Liège, Centre Hospitalier Universitaire \\ Sart Tilman, Liège, Belgium \\ ${ }^{3}$ Department of Nephrology-Dialysis-Apheresis, Hôpital Universitaire Caremeau, Nimes, France
}

\section{ABSTRACT}

Chronic kidney disease (CKD) is defined as abnormalities of kidney structure and function proven to be chronic. The prevalence of CKD in the majority of studies is $10 \%-16 \%$, neglecting the chronicity character. Jonsson et al., in a nationwide study defining CKD adhering strictly to Kidney Disease: Improving Global Outcomes (KDIGO) criteria, found a clearly lower prevalence of CKD (6\%). This indicated that to obtain a correct CKD prevalence, one should start by correctly applying the KDIGO guidelines. 
In 2012, this journal published an update report of the "KDIGO 2012 Clinical Practice Guideline for the Evaluation and Management of Chronic Kidney Disease (CKD)."1 That important paper dramatically changed the existing chaotic approach of the estimation and interpretation of the different CKD classes associated or not with markers of renal damage. ${ }^{2}$

Chronic kidney disease (CKD) is defined as abnormalities of kidney structure (proteinuria, hematuria, renal imaging), present and confirmed within 1 month, with implications for health. Abnormality of kidney function is defined as a glomerular filtration rate (GFR) $<60 \mathrm{ml} / \mathrm{min}$ per $1.73 \mathrm{~m}^{2}$, confirmed at least 3 months after the first determination (chronicity).

Over the past 10 years, a multitude of large and small national and international screening programs, of variable quality, showed that $>10 \%$ up to $16 \%$ of the adult population have markers of CKD stage 1 or 2 or estimated GFR (eGFR) $<60 \mathrm{ml} / \mathrm{min}$ per $1.73 \mathrm{~m}^{2}$ (CKD 3-5), not taking into account the renal damage markers as for CKD 1 and 2.2

One remarkable fact is that the vast majority of these studies neglected to demonstrate the chronicity of the decreased eGFR or the persistence of the increased proteinuria at first test. Bruck et al. ${ }^{3}$ noted "that the prevalence of CKD might have been slightly overestimated using single creatinine and albuminuria measurements. However, this will not have influenced the variation of CKD prevalence across studies. The chronicity criterion was never used, mainly because follow-up data on serum creatinine were not collected."

Several studies illustrated the impact of the ignored chronicity of decreased eGFR on CKD epidemiology. 4 This "slightly overestimated prevalence" turned out to be $22 \%-48 \%$ of the subjects with eGFR $<60 \mathrm{ml} / \mathrm{min}$ per $1.73 \mathrm{~m}^{2}$ at first test, becoming $>60 \mathrm{ml} / \mathrm{min}$ per $1.73 \mathrm{~m}^{2}$ three months later. The percentage of difference between a first test (abnormal) and a control test (normal value) for proteinuria was even more pronounced.

In this issue, Jonsson et al..$^{5}$ bring interesting results of a comprehensive (inclusion of all serum creatinine [sCr] measurements available for individuals $\$ 18$ years of age in the Icelandic population for the entire study period of 2008-2016) nationwide retrospective study of the adult population, defining CKD in strict adherence to the KDIGO criteria. They included 3 different criteria for the definition of CKD: reduced eGFR (chronicity), proteinuria (confirmation), and kidneyspecific diagnosis codes.

They demonstrate a clearly lower prevalence of CKD than most previous studies. The mean annual age-standardized prevalence of CKD according to the full KDIGO criteria was 5.94\% (95\% CI: 5.90-5.98), whereas the prevalence was $12.14 \%$ (95\% CI: 12.08-12.20) when a single value criterion was used. 
Figure 1. The estimated glomerular filtration rate (eGFR) distribution (Modification of Diet in Renal Disease formula) showing the 3rd-97th percentile lines and the age-specific thresholds (red line) in relation to age and sex in the adult population of Morocco. ${ }^{2}$
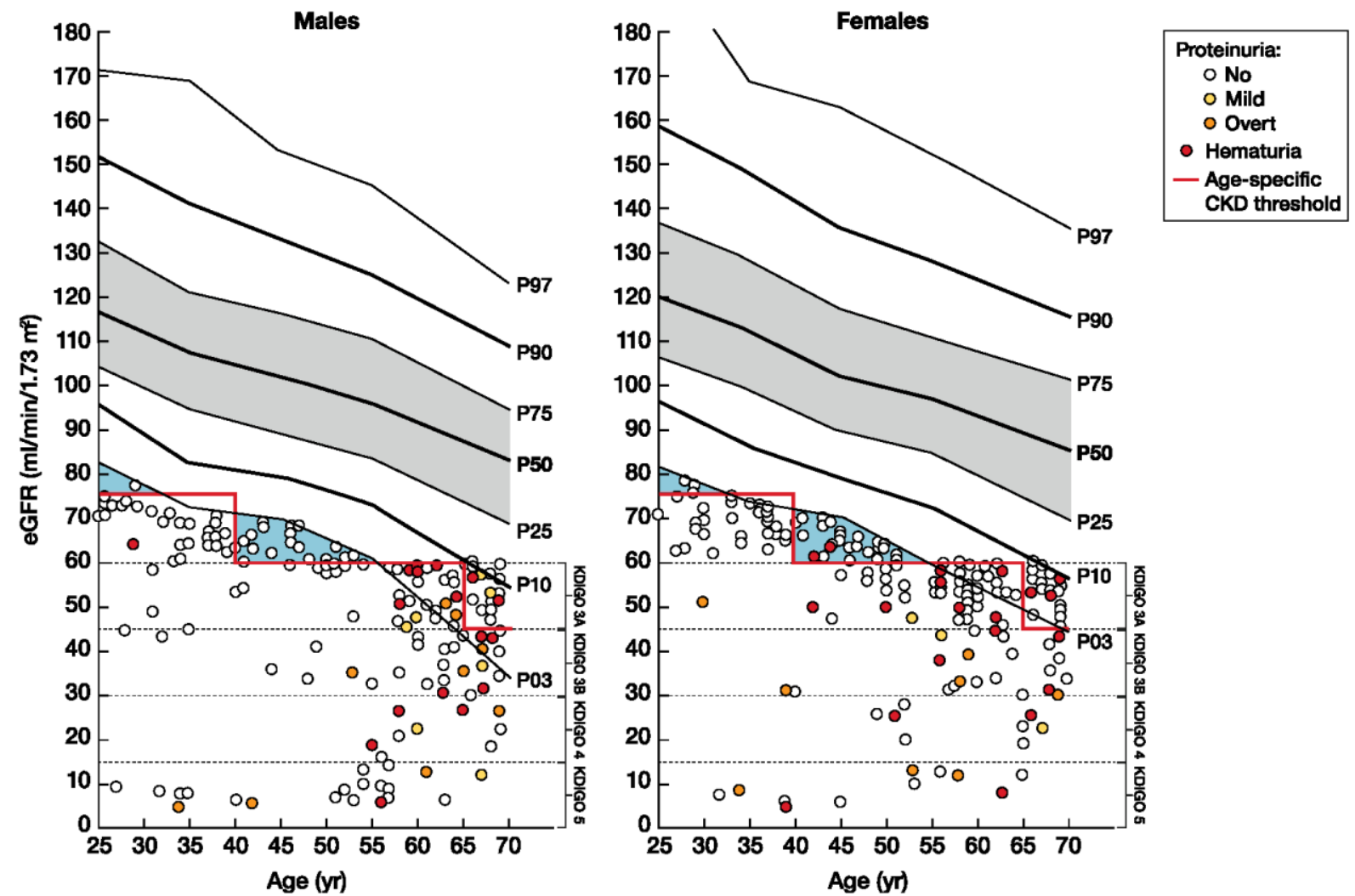

Although the age-specific thresholds clearly decrease the number of "false" results, there remain a number of "false positives and negatives," particularly in the 40-65 years of age category, when comparing these results with the 3rdpercentile line. The latter approach remains the best scientific tool to define the lower eGFR reference limit. CKD, chronic kidney disease; KDIGO, Kidney Disease: Improving Global Outcomes.

Even though they obtained $\mathrm{sCr}$ values from the majority of Icelanders alive during the study period and proteinuria measurements for a large proportion of the population (two-thirds), these data are nonetheless lacking for a limited group of individuals, a shortcoming inherent to retrospective observational studies for estimation of prevalence in the general population that could result in relative falsely low prevalence estimates. However, when they used single eGFR or proteinuria values, prevalence estimates were higher and akin to many previous studies using single measurements, including one from Iceland. This suggests that they may not have missed many individuals with CKD. In addition, they argue that the individuals without sCr are more likely to be healthy or at least healthier than individuals with available sCr levels in this vast study encompassing more than two-thirds of the total population of Iceland.

The MAREMAR (Maladie Renale Chronique au Maroc) study is one of the few prospective randomized (recent voters list) epidemiologic studies of the prevalence of CKD in a representative sample of the adult (26-70 years of age) population, strictly adhering to all the KDIGO guidelines, with a short inclusion period (6 months) and an $85 \%$ response rate. ${ }^{2}$ It is interesting to note that the robust study of Jonsson et al. obtained almost the same low results of 
CKD prevalence as was measured in the MAREMAR study: $\sim 6 \%$ in the Icelandic population and $5.1 \%$ in the Moroccan population. These two comprehensive studies differed in design and ethnicity (Scandinavian, Maghrebian), but both used representative samples of the respective populations and strictly adhered to recent KDIGO guidelines, bringing the prevalence of CKD back to more actual numbers.

In addition to chronicity, there is no evidence that detection of CKD 3A as such in the absence of investigating proteinuria/hematuria in the elderly would be of any diagnostic/therapeutic value. ${ }^{2}$ At least $33 \%$ up to $50 \%$ of the people who meet the definition of $\mathrm{CKD}^{1}$ are classified as stage $3 \mathrm{~A}$.

In an elegant study ${ }^{6}$ in CKD 3 patients, the 10-year cumulative incidence of renal failure (dialysis, transplantation) was 0.04 (95\% CI: 0.03-0.06), in contrast to a mortality incidence of 0.51 (95\% CI: 0.48-0.55), mainly due to cardiovascular diseases. Thus, high mortality apparently preempted the development of renal failure in CKD 3, particularly stage 3A patients. Older patients with CKD 3A, and without proteinuria, have no additional risk of mortality compared with similar-age individuals with an eGFR $>60 \mathrm{ml} / \mathrm{min}$ per $1.73 \mathrm{~m}^{2}$ after adjustment for age, race, sex, and comorbidities.

De Jong and Gansevoort ${ }^{7}$ proposed in 2008 that there is a need to improve the definitions of CKD 3. Confirmed proteinuria should be included in stage 3 before labeling a subject as CKD 3 .

Finally, an arbitrary single threshold of eGFR $<60 \mathrm{ml} / \mathrm{min}$ per $1.73 \mathrm{~m}^{2}$ for classifying CKD 3-5 inevitably leads to overdiagnosis (additional source of false positives) of CKD in the aging group of a population studied, particularly those without proteinuria, hematuria, or overt hypertension who are considered to be healthy older people who will never die of renal failure. ${ }^{8}$ When Jonsson et al. considered age-adapted eGFR thresholds, the age-standardized prevalences of CKD was $3.2 \%$ for men and $4.0 \%$ for women, again clearly lower prevalences of CKD than in the vast majority of previous studies.

Such inaccurate CKD 3A labeling in older individuals without proteinuria/hematuria $(85.9 \%)^{2}$ or hypertension $(61.3 \%)^{2}$ has undesirable effects, such as unnecessary anxiety, unneeded additional investigations, and even loss of insurability.

The single threshold of eGFR $<60 \mathrm{ml} / \mathrm{min}$ per $1.73 \mathrm{~m}^{2}$ also leads to underdiagnosis (false negatives) of CKD in younger individuals with an eGFR $>60 \mathrm{ml} / \mathrm{min}$ per $1.73 \mathrm{~m}^{2}$ and who are below the third percentile of their age/sex category (Figure 1). The use of a third-percentile eGFR level, based on age- and sex-specific reference values of eGFR for a particular population, as cutoff or an age-adapted threshold staging allows the detection of these false positives and negatives. These eGFR curves of many different populations in the world are currently available in the literature. ${ }^{8}$

Although these simple concepts have been supported for several years by solid publications in the best journals of medicine and nephrology, the scientific renal community has not arrived at a consensus regarding the interpretation of a particular eGFR and its clinical consequences. However, some light is appearing at the end of the tunnel. recently

The Global Burden of Disease Chronic Kidney Disease Collaboration wrote: 9 "Most data sources reporting the prevalence of nonfatal CKD are cross sectional and do not repeat serum creatinine 
and urine ACR measurements over 3 months, as suggested by KDIGO guidelines, to confirm the chronicity of abnormalities. Studies suggest that use of one measurement of decreased eGFR to characterize CKD might overestimate prevalence by $25 \%$ - 50\%. Therefore, it is possible that the results of our analysis represent an overestimate of CKD prevalence. Future analyses of the global burden of CKD should investigate developing a methodology to correct prevalence estimates."

Jonsson et al. have added new relevant data contributing to the realization of the above-defined wish in the near future.

\section{DISCLOSURE}

All the authors declared no competing interests. 


\section{References}

1. Kidney Disease: Improving Global Outcomes (KDIGO) CKD Work Group. KDIGO 2012 clinical practice guideline for the evaluation and management of chronic kidney disease. Kidney Int Suppl. 2013;3:1-150.

2. Bengahnem Gharbi M, Elseviers M, et al. Chronic kidney disease, hypertension, diabetes, and obesity in the adult population of Morocco: how to avoid "over" and "under" diagnosis of CKD. Kidney Int. 2016;89:1363- 1371.

3. Brück K, Stel VS, Gambaro G, et al. CKD prevalence varies across the European general population. J Am Soc Nephrol. 2015;27:2135-2147.

4. Delanaye P. Too much nephrology? The CKD epidemic is real and concerning. A CON view. Nephrol Dial Transplant. 2019;34:581- 584.

5. Jonsson AJ, Lund SH, Eriksen BO, et al. The prevalence of chronic kidney disease in Iceland according to KDIGO criteria and age- adapted estimated glomerular filtration rate thresholds. Kidney Int. 2020;98:1286-1295.

6. Eriksen BO, Ingebretsen OC. The progression of chronic kidney disease: a 10-year population-based study of the effects of gender and age. Kidney Int. 2006;69:375-382.

7. de Jong PE, Gansevoort RT. Fact or fiction of the epidemic of chronic kidney disease-let us not squabble about estimated GFR only, but also focus on albuminuria. Nephrol Dial Transplant. 2008;23:1092-1095.

8. Delanaye P, Jager KJ, Bolkenkamp A, et al. CKD: a call for an age-adapted definition. JAm Soc Nephrol. 2019;30:1785-1805.

9. GBD Chronic Kidney Disease Collaboration. Global, regional, and national burden of chronic kidney disease, 1990-2017: a systematic analysis for the Global Burden of Disease Study 2017. Lancet 395(10225):709- 733. 